\title{
Selective amidation of unprotected amino-alcohols using Surfactant-in-Water Technology: A highly desirable alternative to reprotoxic polar aprotic solvents.
}

\author{
Michael Parmentier ${ }^{*},{ }^{,}$, Mona K. Wagner, ${ }^{\ddagger}$ Kevin Magra, ${ }^{\ddagger}$ Fabrice Gallou ${ }^{+}$ \\ Chemical \& Analytical Development, Novartis Pharma AG, 4056 Basel, Switzerland
}

\section{Supporting Information}

\section{Contents}

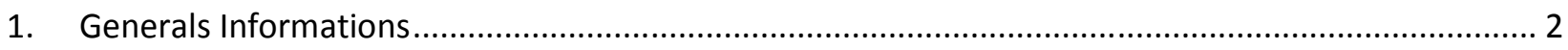

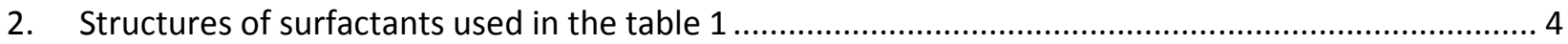

3. Synthesis of by-products derived from amidation of 4-bromobenzoic acid (1) and 2-amino-2-

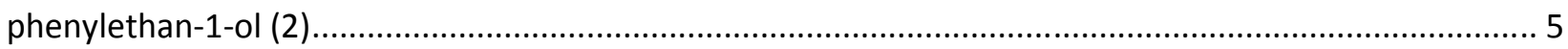

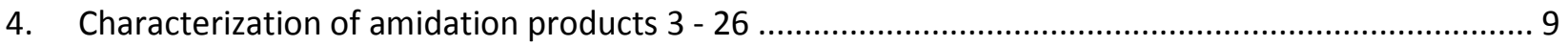

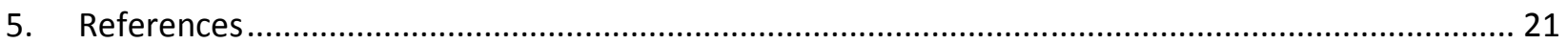




\section{General Informations}

A solution of $2 \mathrm{wt} \%$ TPGS-750-M/ $\mathrm{H}_{2} \mathrm{O}$ was prepared by dissolving TPGS-750-M in deionized water. All commercially available reagents were used without further purification including TPGS-750-M which was purchased from Anthem Biosciences (CAS-No. 1309573-60-1).

${ }^{1} \mathrm{H}$ and ${ }^{13} \mathrm{C}$ NMR were recorded at $297.8 \mathrm{~K}$ on a Bruker ${ }^{\circledR} 400 \mathrm{MHz}$ spectrometer. The FID was processesed using MestReNova NMR analysis software. Chemical shifts in ${ }^{1} \mathrm{H}$ NMR spectra are reported in parts per million (ppm) on the $\delta$ scale from an internal standard of residual $\mathrm{CDCl}_{3}$ $(7.260 \mathrm{ppm})$ or the central peak of DMSO- $d_{6}(2.50 \mathrm{ppm})$. Data are reported as follows: chemical shift, multiplicity ( $s=$ singlet, $d=$ doublet, $t=$ triplet, $q=$ quartet, quin = quintet), and integration. Chemical shifts in ${ }^{13} \mathrm{C}$ chemical spectra are reported in ppm on the $\delta$ scale from the central peak of residual $\mathrm{CDCl}_{3}(77.16 \mathrm{ppm})$ or the central peak of DMSO- $d_{6}(39.51 \mathrm{ppm})$.

Reaction profile based on analysis of UPLC/MS data using Acquity HSS T3 $1.8 \mu \mathrm{m} 2.1$ x 50 mm at $60^{\circ} \mathrm{C}$ with the following eluent system: (A: water $+0.05 \%$ formic acid $+3.75 \mathrm{mM}$ ammonium acetate; B: acetonitrile $+0.04 \%$ formic acid); gradient from 5 to $98 \%$ B in 1.4 min; flow 1.0 $\mathrm{mL} / \mathrm{min}$.

\section{General procedure A for an amide-bond forming reaction in TPGS-750-M/water}

To a mixture of carboxylic acid (2.50 mmol, $1.0 \mathrm{eq})$, EDC (0.715 g, $3.73 \mathrm{mmol}, 1.5 \mathrm{eq})$, HOBt (0.457 g, $3.0 \mathrm{mmol}, 1.2 \mathrm{eq})$, aminoalcohol (3.0 mmol, $1.2 \mathrm{eq})$ and NMM (0.82 mL, $7.46 \mathrm{mmol}$, 3.0 eq) was added TPGS-750-M (2 wt\% in water, $10 \mathrm{~mL})$. The reaction was allowed to stir for 16-24 hours at $40{ }^{\circ} \mathrm{C}$, until no starting material was detected. The reaction progress was followed by LCMS.

The suspension was cooled to RT, filtered, washed with water $(2 \times 5.0 \mathrm{~mL})$ and dried at $50{ }^{\circ} \mathrm{C}$ under reduced pressure for 12 hours. The resulting product was analyzed by NMR-spectroscopy and HRMS-spectrometry.

\section{General procedure B for an amide-bond forming reaction in acetonitrile or DMF}

To a mixture of carboxylic acid (2.50 mmol, $1.0 \mathrm{eq})$, EDC (0.715 g, $3.73 \mathrm{mmol}, 1.5 \mathrm{eq})$, HOBt (0.457 g, $3.0 \mathrm{mmol}, 1.2 \mathrm{eq})$, aminoalcohol (3.0 mmol, $1.2 \mathrm{eq})$ and NMM (0.82 mL, $7.46 \mathrm{mmol}$, $3.0 \mathrm{eq})$ was added acetonitrile or DMF $(10 \mathrm{~mL})$. The reaction was allowed to stir for $16-24$ hours 
at room temperature, until no starting material was left. The reaction progress was followed by LCMS.

The solution was diluted with saturated $\mathrm{NaHCO}_{3}(10 \mathrm{~mL})$ and EtOAc $(20 \mathrm{~mL})$. The phases were allowed to separate and the water phase was extracted with further EtOAc $(2 \mathrm{x} 10 \mathrm{~mL})$. The combined organic layers were washed with water $(25 \mathrm{~mL})$ to remove further HOBt. The water phase was extracted once with EtOAc $(25 \mathrm{~mL})$. The combined organic layers were dried over $\mathrm{Na}_{2} \mathrm{SO}_{4}$, filtrated and concentrated under reduced pressure. The resulting product was analyzed by NMR-spectroscopy and HRMS-spectrometry.

\section{General procedure $C$ for an amide-bond forming reaction in TPGS-750-M/water requiring extractive workup}

To a mixture of carboxylic acid $(2.50 \mathrm{mmol}, 1.0 \mathrm{eq})$, EDC (0.715 g, $3.73 \mathrm{mmol}, 1.5 \mathrm{eq})$, HOBt (0.457 g, $3.0 \mathrm{mmol}, 1.2 \mathrm{eq})$, aminoalcohol (3.0 mmol, $1.2 \mathrm{eq})$ and NMM (0.82 mL, $7.46 \mathrm{mmol}$, $3.0 \mathrm{eq})$ was added TPGS-750-M (2 wt $\%$ in water, $10 \mathrm{~mL})$. The reaction was allowed to stir for 16-24 hours at $40{ }^{\circ} \mathrm{C}$, until no starting material was left. The reaction progress was followed by LCMS.

The solution was diluted with saturated $\mathrm{NaHCO}_{3}(10 \mathrm{~mL})$ and EtOAc $(20 \mathrm{~mL})$. The phases were allowed to separate and the water phase was extracted with further EtOAc $(2 \times 10 \mathrm{~mL})$. The combined organic layers were washed with water $(25 \mathrm{~mL})$ to remove further HOBt. The water phase was extracted once with EtOAc $(25 \mathrm{~mL})$. The combined organic layers were dried over $\mathrm{Na}_{2} \mathrm{SO}_{4}$, filtrated and concentrated under reduced pressure. The resulting product was analyzed by NMR-spectroscopy and HRMS-spectrometry. 


\section{Structures of surfactants used in the table 1}

\section{TWEEN 80 :}

$\mathrm{HO}_{\mathrm{w}}\left(\mathrm{H}_{2} \mathrm{CH}_{2} \mathrm{CO}\right)$

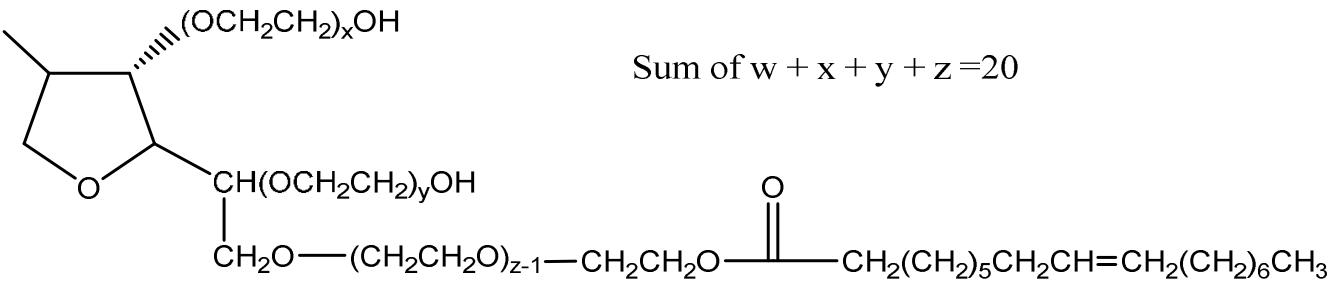

NOK :<smiles>CC[C@H](CC[C@@H](C)[C@H]1CC[C@H]2[C@@]3(C)CC=C4CC(OC(=O)CCC(=O)OCC(C)COCC(C)(C)OC)CC[C@]4(C)[C@H]3CC[C@]12C)C(C)C</smiles>

$n \sim 13$

\section{SOLUTOL-HS :}<smiles>CCCCCCCCC(=O)C(C)OCCC(C)(C)O</smiles>

TPGS-1000 :<smiles>Cc1c(C)c2c(c(C)c1OC(=O)CCC(=O)OCCOc1ccccc1)CCC(C)(CCCC(C)(C)C)O2</smiles> 


\section{Synthesis of by-products derived from amidation of 4- bromobenzoic acid (1) and 2-amino-2-phenylethan-1-ol (2)}<smiles>CC(C(=O)OCC(NC(=O)OC(C)(C)C)c1ccccc1)C(C)c1ccccc1C(CO)NC(=O)OC(C)(C)C</smiles><smiles>CC(COC(=O)c1ccc(Br)cc1)c1ccccc1</smiles>

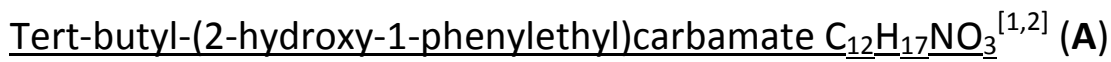<smiles>CC(C)(C)OC(=O)NC(CO)c1ccccc1</smiles>

Di-tert-butyl dicarbonate $(0.875 \mathrm{~g}, 4.0 \mathrm{mmol}, 1.1 \mathrm{eq})$ and sulfamic acid $(0.018 \mathrm{~g}, 0.182 \mathrm{mmol}$, $0.05 \mathrm{eq})$ were stirred together at $30^{\circ} \mathrm{C}$. 2-amino-2-phenylethanol $(0.5 \mathrm{~g}, 3.65 \mathrm{mmol}, 1.0 \mathrm{eq})$ was added to the colourless solution under heavy gas formation. A mixture of acetonitrile : water (1 : 1, $6 \mathrm{~mL}$ ) was added to the white solid and the suspension was stirred for 30 minutes. The mixture was filtered and the white residue was dried at $50{ }^{\circ} \mathrm{C}$ under reduced pressure for 16 hours. Tertbutyl-(2-hydroxy-1-phenylethyl)carbamate was obtained as a white solid in $84 \%$ yield.

$\mathbf{M p}=137.7-138.8^{\circ} \mathrm{C}$. 
${ }^{1}$ H-NMR (400 MHz, DMSO-d $\left.d_{6}\right) \delta=7.30-7.27$ (m, 4H, $\left.H_{\text {arom. }}\right), 7.23-7.17$ (m, 2H, $H_{\text {arom., }} \mathrm{N} H$ ), $4.76(\mathrm{t}, J=5.8 \mathrm{~Hz}, 1 \mathrm{H}, \mathrm{OH}), 4.55-4.49(\mathrm{~m}, 1 \mathrm{H}, \mathrm{CHNH}), 3.48$ (t, $\left.J=6.3 \mathrm{~Hz}, 2 \mathrm{H}, \mathrm{CH}_{2}\right), 1.37$ (s, $\left.9 \mathrm{H}, 3 \times \mathrm{CH}_{3}\right) \mathrm{ppm}$.

${ }^{13}$ C-NMR $\left(100 \mathrm{MHz}, \mathrm{DMSO}-d_{6}\right) \delta=155.17(\mathrm{C}=\mathrm{O}), 141.8,128.0,128.0,126.8,126.8,126.6(6 \mathrm{x}$ $\left.\mathrm{C}_{\text {arom }}\right), 77.7(\mathrm{C}), 64.8(\mathrm{C}), 56.8(\mathrm{C}), 28.2(\mathrm{C}) \mathrm{ppm}$.

HRMS (ESI) $m / z:(\mathrm{M}+\mathrm{H})^{+}$calc. for $\mathrm{C}_{12} \mathrm{H}_{17} \mathrm{NO}_{3}, 237.1365$ found 237.1438 .

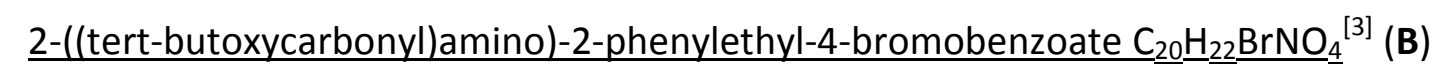<smiles>CC(C)(C)OC(=O)NC(COC(=O)c1ccc(Br)cc1)c1ccccc1</smiles>

tert-butyl (2-hydroxy-1-phenylethyl)carbamate $\quad(0.237 \mathrm{~g}, \quad 1.0 \mathrm{mmol}, \quad 0.7 \mathrm{eq}), \quad$ N1,N1,N2,N2tetramethylethane-1,2-diamine $(0.23 \mathrm{~mL}, 1.5 \mathrm{mmol}, 1.0 \mathrm{eq})$ and 1-methyl- $1 H$-imidazole $(0.12$ $\mathrm{mL}, 1.5 \mathrm{mmol}, 1.0 \mathrm{eq})$ were diluted in acetonitrile $(4.0 \mathrm{~mL})$ under inert-gas-atmosphere. The white suspension was cooled to $0{ }^{\circ} \mathrm{C}$ and 4-bromobenzoyl chloride $(0.329 \mathrm{~g}, 1.5 \mathrm{mmol}, 1.0 \mathrm{eq})$ was added. The white suspension was allowed to stir $3 \mathrm{~h}$ at $0^{\circ} \mathrm{C}$ and further 16 hours at ambient temperature. The mixture was filtered, washed with water and the residue was dried at $50{ }^{\circ} \mathrm{C}$ under reduced pressure for 16 hours. (2-((tert-butoxycarbonyl)amino)-2-phenylethyl-4bromobenzoate was obtained as a white solid in 54\% yield.

$\mathbf{M p}=99.5-101^{\circ} \mathrm{C}$.

${ }^{1}$ H-NMR $\left(400 \mathrm{MHz}, \mathrm{DMSO}_{\mathrm{d}}\right) \delta=7.87\left(\mathrm{~d}, J=8.6 \mathrm{~Hz}, 2 \mathrm{H}, H_{\text {arom }}\right), 7.75(\mathrm{~d}, 2 \mathrm{H}, J=8.4 \mathrm{~Hz}$, $\left.H_{\text {arom }}\right), 7.71(\mathrm{~d}, J=9.1 \mathrm{~Hz}, 1 \mathrm{H}, \mathrm{NH}), 7.43$ (d, 2H, $\left.J=7.4 \mathrm{~Hz}, H_{\text {arom }}\right), 7.36$ (t, $J=7.4 \mathrm{~Hz}, 2 \mathrm{H}$, $\left.H_{\text {arom }}\right), 7.29-7.23\left(\mathrm{~m}, 1 \mathrm{H}, H_{\text {arom }}\right), 5.03(\mathrm{~m}, 1 \mathrm{H}, \mathrm{CH}), 4.40\left(\mathrm{dd}, 1 \mathrm{H}, \mathrm{CH}_{2}\right), 4.33$ (dd, $\left.1 \mathrm{H}, \mathrm{CH}_{2}\right)$, $1.36\left(\mathrm{~s}, 9 \mathrm{H}, \mathrm{CH}_{3}\right) \mathrm{ppm}$.

${ }^{13}$ C-NMR $\left(100 \mathrm{MHz}, \mathrm{DMSO}-d_{6}\right) \delta=186.3(\mathrm{COONH}), 165.3(\mathrm{COO}), 150.2\left(\mathrm{CO}\left(\mathrm{CH}_{3}\right)_{3}\right), 132.3$, $132.3,131.6,131.6,129.2,128.8,128.8,128.0,127.9,127.4,127.4,110.0\left(C_{\text {arom }}\right), 78.6\left(\mathrm{COH}_{2}\right)$, 53.5, (CHN), $28.6(3 \times \mathrm{C}) \mathrm{ppm}$. 
HRMS (ESI) $m / z:(\mathrm{M}+\mathrm{H})^{+}$calc. for $\mathrm{C}_{20} \mathrm{H}_{22} \mathrm{BrNO}_{4}, 419.0732$ found 419.0805 .

2-amino-2-phenylethyl-4-bromobenzoate ${ }^{[4]}$ (C)<smiles>NC(COC(=O)c1ccc(Br)cc1)c1ccccc1</smiles>

2-((tert-butoxycarbonyl)amino)-2-phenylethyl-4-bromobenzoate $(0.2 \mathrm{~g}, 0.476 \mathrm{mmol})$ was diluted in $\mathrm{CH}_{2} \mathrm{Cl}_{2}(0.7 \mathrm{~mL})$ and Trifluoroacetic acid $(0.7 \mathrm{~mL})$ was added. After 2 hours at ambient temperature, the excess of $\mathrm{CH}_{2} \mathrm{Cl}_{2}$ and Trifluoroacetic acid was removed under reduced pressure. The resulting yellow oil was diluted in $\mathrm{NaHCO}_{3}(3.0 \mathrm{~mL})$ and $\mathrm{CH}_{2} \mathrm{Cl}_{2}(5.0 \mathrm{~mL})$. The phases were allowed to separate and the water phase was extracted with further $\mathrm{CH}_{2} \mathrm{Cl}_{2}(2 \times 5.0 \mathrm{~mL})$. The combined organic phases were dried over $\mathrm{Na}_{2} \mathrm{SO}_{4}$, filtered and the solvent was removed under reduced pressure. 2-amino-2-phenylethyl 4-bromobenzoate was obtained as a yellow solid in $85 \%$ yield.

$\mathbf{M p}=141.5-143.5^{\circ} \mathrm{C}$.

${ }^{1}$ H-NMR (400 MHz, DMSO-d6) $\delta=8.77(\mathrm{~s}, 2 \mathrm{H}, \mathrm{N} H), 8.02\left(\mathrm{~d}, J=8.7 \mathrm{~Hz}, 2 \mathrm{H}, H_{\text {arom }}\right), 7.79(\mathrm{~d}, J$ $\left.=8.7 \mathrm{~Hz}, 2 \mathrm{H}, H_{\text {arom }}\right), 7.58\left(\mathrm{~d}, J=7.2 \mathrm{~Hz}, 2 \mathrm{H}, H_{\text {arom }}\right), 7.50-7.44\left(\mathrm{~m}, 3 \mathrm{H}, H_{\text {arom }}\right), 4.83(\mathrm{t}, J=6$ $\mathrm{Hz}, 1 \mathrm{H}, \mathrm{CH}), 4.58\left(\mathrm{~d}, J=6 \mathrm{~Hz}, 2 \mathrm{H}, \mathrm{CH}_{2}\right) \mathrm{ppm}$.

${ }^{13}$ C-NMR (100 MHz, DMSO-d6) $\delta=165.1(C=0), 134.9,132.3,132.3,132.1,129.6,129.4$, 129.4, 128.6, 128.4, 127.9, 127.9 (12x $\left.C_{\text {arom }}\right), 70.2(\mathrm{COH}), 65.9\left(\mathrm{CNH}_{2}\right) \mathrm{ppm}$.

HRMS (ESI) $m / z:(\mathrm{M}+\mathrm{H})^{+}$calc. for $\mathrm{C}_{15} \mathrm{H}_{14} \mathrm{BrNO}_{2}, 319.0208$ found 319.0281 .

(S)-2-(4-bromobenzamido)-2-phenylethyl-4-bromobenzoate $\underline{\mathrm{C}}_{22} \underline{\mathrm{H}}_{17} \underline{\mathrm{Br}}_{2} \underline{\mathrm{NO}}_{3}{ }^{[3]} \mathbf{3 b}$<smiles>O=C(NC(COC(=O)c1ccc(Br)cc1)c1ccccc1)c1ccc(Br)cc1</smiles> 
(S)-4-bromo-N-(2-hydroxy-1-phenylethyl)benzamide ( $0.320 \mathrm{~g}, 1.0 \mathrm{mmol}, 0.7 \mathrm{eq}), \mathrm{N1}, \mathrm{N1}$,N2,N2tetramethylethane-1,2-diamine $(0.23 \mathrm{~mL}, 1.5 \mathrm{mmol}, 1.0 \mathrm{eq})$ and 1-methyl- $1 H$-imidazole $(0.12$ $\mathrm{mL}, 1.5 \mathrm{mmol}, 1.0 \mathrm{eq})$ were diluted in acetonitrile $(4.0 \mathrm{~mL})$ under inert-gas-atmosphere. The white suspension was cooled to $0{ }^{\circ} \mathrm{C}$ and 4-bromobenzoyl chloride $(0.329 \mathrm{~g}, 1.5 \mathrm{mmol}, 1.0 \mathrm{eq})$ was added. The white suspension was allowed to stir 3 hours at $0^{\circ} \mathrm{C}$ and further 16 hours at ambient temperature. The mixture was filtered, washed with water and the residue was dried at $50{ }^{\circ} \mathrm{C}$ under reduced pressure for 16 hours. (S)-2-(4-bromobenzamido)-2-phenylethyl-4bromobenzoate was obtained as a white solid in $54 \%$ yield.

$\mathbf{M p}=198-200{ }^{\circ} \mathrm{C}$.

${ }^{1}$ H-NMR $\left(400 \mathrm{MHz}\right.$, DMSO- $\left.d_{6}\right) \delta=9.14(\mathrm{~d}, J=8.3 \mathrm{~Hz}, 1 \mathrm{H}, \mathrm{N} H), 7.82(\mathrm{~d}, \mathrm{~J}=8.3 \mathrm{~Hz}, 4 \mathrm{H}$, $\left.H_{\text {arom }}\right), 7.73-7.68\left(\mathrm{~m}, 4 \mathrm{H}, H_{\text {arom. }}\right), 7.51\left(\mathrm{~d}, J=7.3 \mathrm{~Hz}, 2 \mathrm{H}, H_{\text {arom. }}\right), 7.38$ (t, $J=7.4 \mathrm{~Hz}, 2 \mathrm{H}$, $\left.H_{\text {arom }}\right), 7.31-7.28\left(\mathrm{~m}, 1 \mathrm{H}, H_{\text {arom }}\right), 5.54$ (q, $\left.J=7.2 \mathrm{~Hz}, 1 \mathrm{H}, \mathrm{C} H \mathrm{NH}\right), 4.57(\mathrm{~d}, J=7.0 \mathrm{~Hz}, 2 \mathrm{H}$, $\left.\mathrm{CH}_{2}\right) \mathrm{ppm}$.

${ }^{13}$ C-NMR $\left(100 \mathrm{MHz}\right.$, DMSO- $\left.d_{6}\right) \delta=165.4(\mathrm{C}=\mathrm{O}), 164.9(\mathrm{C}=\mathrm{O}), 139.2,133.4,131.9,131.9$, $131.3,131.3,131.0,131.0,129.5,129.5,128.7,128.5,128.5,127.6,127.0,127.0,125.1,109.5$ (18 x C $\left.\mathrm{C}_{\text {arom }}\right), 66.7(\mathrm{C}), 52.1$ (C) ppm.

HRMS (ESI) $m / z:(\mathrm{M}+\mathrm{H})^{+}$calc. for $\mathrm{C}_{22} \mathrm{H}_{17} \mathrm{Br}_{2} \mathrm{NO}_{3}$ 501.9648, found 501.9653 . 


\section{Characterization of amidation products $3-26$}

(S) 4-bromo- $N$-(2-hydroxy-1-phenylethyl)benzamide $\mathrm{C}_{15} \underline{\mathrm{H}_{14}} \underline{\mathrm{BrNO}}_{2} 3$<smiles>O=C(NC(CO)c1ccccc1)c1ccc(Br)cc1</smiles>

The titled compound was obtained with general procedure A in 95\% yield, using general procedure B in 92\% yield (acetonitrile) and 99\% yield (DMF).

$\mathbf{M p}=178-179.5^{\circ} \mathrm{C}$.

${ }^{1}$ H-NMR $\left(400 \mathrm{MHz}, \mathrm{DMSO}-d_{6}\right) \delta=8.80(\mathrm{~d}, J=8.1 \mathrm{~Hz}, 1 \mathrm{H}, \mathrm{NH}), 7.85(\mathrm{~d}, J=8.6 \mathrm{~Hz}, 2 \mathrm{H}$, $\left.H_{\text {arom }}\right), 7.69$ (d, $\left.J=8.6 \mathrm{~Hz}, 2 \mathrm{H}, H_{\text {arom }}\right), 7.39-7.37$ (m, 2H, $\left.H_{\text {arom }}\right), 7.33-7.29$ (m, $\left.2 \mathrm{H}, H_{\text {arom }}\right)$, $7.25-7.21\left(\mathrm{~m}, 1 \mathrm{H}, H_{\text {arom }}\right), 5.02-5.08(\mathrm{~m}, 1 \mathrm{H}, \mathrm{CHNH}), 4.95(\mathrm{t}, J=5.9 \mathrm{~Hz}, 1 \mathrm{H}, \mathrm{OH}), 3.73-3.60$ $\left(\mathrm{m}, 2 \mathrm{H}, \mathrm{CH}_{2}\right) \mathrm{ppm}$.

${ }^{13}$ C-NMR $\left(100 \mathrm{MHz}, \mathrm{DMSO}-d_{6}\right) \delta=165.2(\mathrm{C}=\mathrm{O}), 141.2,133.7,131.2,131.2,129.6,129.6$, $128.1,128.1,127.0,127.0,126.9,124.9,\left(12 \times \mathrm{C}_{\text {arom }}\right), 64.5(\mathrm{C}), 56.1(\mathrm{C}) \mathrm{ppm}$.

HRMS (ESI) $m / z:(\mathrm{M}+\mathrm{H})^{+}$calc. for $\mathrm{C}_{16} \mathrm{H}_{14} \mathrm{BrNO}_{2} 320.0281$, found 320.0284 .

Protocole : To a $250 \mathrm{~mL}$ reactor was added 4-bromobenzoic acid (10 g, $49.7 \mathrm{mmol}$, 1eq), TPGS750-M (100 mL), HOBt (8.07 g, 59.7 mmol, 1.2 eq), EDC (14.31 g, 74.6 mmol, 1.5 eq), (S)-2amino-2-phenylethanol (8.19 g, $59.7 \mathrm{mmol}, 1.2 \mathrm{eq})$, NMM (16.4 mL, $149.2 \mathrm{mmol}, 3 \mathrm{eq})$ and the remaining TPGS-750-M (50 mL). Then the mixture was heated to $40^{\circ} \mathrm{C}$ and stirred during 17 hours at $40^{\circ} \mathrm{C}$. After, the reaction mixture was cooled to $20^{\circ} \mathrm{C}$ and filtered on fritten who the product was washed with water $(2 \times 75 \mathrm{~mL})$. The powder was dried at $50^{\circ} \mathrm{C}$ under reduced pressure. (S) 4-bromo- $N$-(2-hydroxy-1-phenylethyl)benzamide was obtiened as a white solid in $95 \%$ yield.

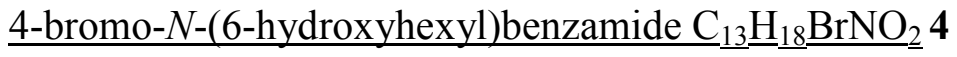




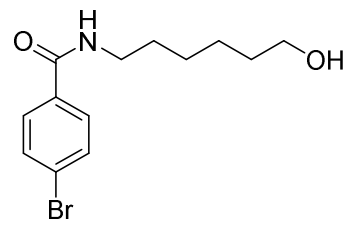

The titled compound was obtained with general procedure A in 93\% yield, using general procedure $\mathrm{B}$ in $85 \%$ yield (acetonitrile) and $88 \%$ yield (DMF).

$\mathbf{M p}=117-118^{\circ} \mathrm{C}$.

${ }^{1}$ H-NMR $\left(400 \mathrm{MHz}, \mathrm{DMSO}-d_{6}\right) \delta=8.49(\mathrm{t}, J=5.2 \mathrm{~Hz}, 1 \mathrm{H}, \mathrm{N} H), 7.78\left(\mathrm{~d}, J=8.8 \mathrm{~Hz}, 2 \mathrm{H}, H_{\text {arom}}\right)$, $7.66\left(\mathrm{~d}, J=8.8 \mathrm{~Hz}, 2 \mathrm{H}, H_{\text {arom }}\right), 4.31(\mathrm{t}, J=5.2 \mathrm{~Hz}, 1 \mathrm{H}, \mathrm{OH}), 3.37\left(\mathrm{q}, J=6.5 \mathrm{~Hz}, 2 \mathrm{H}, \mathrm{CH}_{2}\right), 3.23$ $\left(\mathrm{q}, J=6.5 \mathrm{~Hz}, 2 \mathrm{H}, \mathrm{CH}_{2}\right), 1.53-1.28\left(\mathrm{~m}, 8 \mathrm{H}, 4 \mathrm{x} \mathrm{CH} \mathrm{CH}_{2}\right) \mathrm{ppm}$.

${ }^{13}$ C-NMR $\left(100 \mathrm{MHz}, \mathrm{DMSO}-d_{6}\right) \delta=165.1(\mathrm{C}=\mathrm{O}), 133.8,131.2,131.2,129.2,129.2,124.6(6 \mathrm{x}$ $\mathrm{C}_{\text {arom }}$ ), $60.6(\mathrm{C}), 39.2$ (C), 32.4 (C), 29.0 (C) 26.4 (C), 25.2 (C) ppm.

HRMS (ESI) $m / z:(\mathrm{M}+\mathrm{H})^{+}$calc. for $\mathrm{C}_{13} \mathrm{H}_{18} \mathrm{BrNO}_{2} 300.0594$, found 300.0593 .

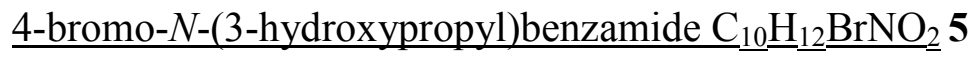<smiles>O=C(NCCCO)c1ccc(Br)cc1</smiles>

The titled compound was obtained with general procedure A in $91 \%$ yield, using general procedure B in $75 \%$ yield (acetonitrile) and 94\% yield (DMF).

$\mathbf{M p}=71.5-73{ }^{\circ} \mathrm{C}$.

${ }^{1}$ H-NMR (400 MHz, DMSO- $\left.d_{6}\right) \delta=8.50(\mathrm{t}, J=5.2 \mathrm{~Hz}, 1 \mathrm{H}, \mathrm{N} H), 7.79-7.77\left(\mathrm{~m}, 2 \mathrm{H}, H_{\text {arom }}\right)$, $7.67-7.65\left(\mathrm{~m}, 2 \mathrm{H}, H_{\text {arom }}\right), 4.45(\mathrm{t}, J=5.2 \mathrm{~Hz}, 1 \mathrm{H}, \mathrm{OH}), 3.46\left(\mathrm{q}, J=6.2 \mathrm{~Hz}, 5.2 \mathrm{~Hz}, 2 \mathrm{H}, \mathrm{CH}_{2}\right)$, $3.28-3.33\left(\mathrm{~m}, 2 \mathrm{H}, \mathrm{CH}_{2}\right), 1.67$ (qi, $J=6.5 \mathrm{~Hz}, 2 \mathrm{H}, \mathrm{CH}_{2} \mathrm{CH}_{2} \mathrm{CH}_{2}$ ) ppm.

${ }^{13}$ C-NMR $\left(100 \mathrm{MHz}, \mathrm{DMSO}-d_{6}\right) \delta=165.2(\mathrm{C}=\mathrm{O}), 133.7,131.2,131.2,129.2,129.2,124.7(6 \mathrm{x}$ $\left.\mathrm{C}_{\text {arom }}\right), 58.6(C), 36.6(\mathrm{C}), 32.3(\mathrm{C}) \mathrm{ppm}$.

HRMS (ESI) $m / z:(\mathrm{M}+\mathrm{H})^{+}$calc. for $\mathrm{C}_{10} \mathrm{H}_{12} \mathrm{BrNO}_{2} 258.0124$, found 258.0123.

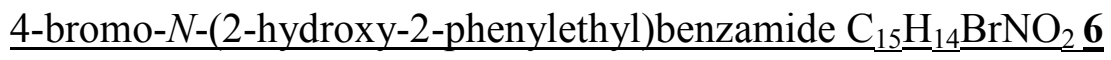




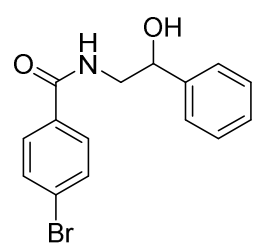

The titled compound was obtained with general procedure A in $90 \%$ yield, using general procedure B in 94\% yield (acetonitrile) and 86\% yield (DMF).

$\mathbf{M p}=71-73^{\circ} \mathrm{C}$.

${ }^{1}$ H-NMR (400 MHz, DMSO- $\left.d_{6}\right) \delta=8.62(\mathrm{t}, J=5.5 \mathrm{~Hz}, 1 \mathrm{H}, \mathrm{N} H), 7.79\left(\mathrm{~d}, J=8.6 \mathrm{~Hz}, 2 \mathrm{H}, H_{\text {arom}}\right)$, $7.67\left(\mathrm{~d}, J=8.5 \mathrm{~Hz}, 2 \mathrm{H}, H_{\text {arom }}\right), 7.38-7.31\left(\mathrm{~m}, 4 \mathrm{H}, H_{\text {arom }}\right), 7.27-7.21\left(\mathrm{~m}, 1 \mathrm{H}, H_{\text {arom }}\right), 5.52(\mathrm{~d}, J$ $=4.4 \mathrm{~Hz}, 1 \mathrm{H}, \mathrm{OH}), 4.80-4.76(\mathrm{~m}, 1 \mathrm{H}, \mathrm{CHOH}), 3.51-3.45\left(\mathrm{~m}, 1 \mathrm{H}, \mathrm{CH}_{2}\right), 3.35-3.29(\mathrm{~m}, 1 \mathrm{H}$, $\left.\mathrm{CH}_{2}\right) \mathrm{ppm}$.

${ }^{13}$ C-NMR (100 MHz, DMSO- $\left.d_{6}\right) \delta=165.4(\mathrm{C}=\mathrm{O}), 143.7,133.7,131.2,131.2,129.4,129.4$, 128.0, 128.0, 127.0, 126.0, 126.0, 124.8, (12x C $\mathrm{arom}_{\text {) }} 71.1(\mathrm{C}), 47.7$ (C) ppm.

HRMS (ESI) $m / z:(\mathrm{M}+\mathrm{H})^{+}$calc. for $\mathrm{C}_{15} \mathrm{H}_{14} \mathrm{BrNO}_{2} 320.0281$, found 320.0283 .

Comparison: A. S. Aboraia, S. W. Yee, M. S. Gomaa, N. Shah, A. C. Robotham, B. Makowski, D. Prosser, A. Brancale, G. Jones, C. Simons, Bioorg. Med. Chem. 2010, 18, 4939-4946.

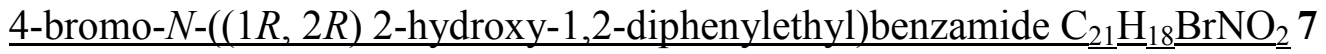<smiles>O=C(NC(c1ccccc1)C(O)c1ccccc1)c1ccc(Br)cc1</smiles>

The titled compound was obtained with general procedure A in $82 \%$ yield, using general procedure B in $88 \%$ yield (acetonitrile) and $87 \%$ yield (DMF).

$\mathbf{M p}=235-237^{\circ} \mathrm{C}$.

${ }^{1}$ H-NMR (400 MHz, DMSO-d $\left.{ }_{6}\right) \delta=8.71(\mathrm{~d}, \mathrm{~J}=9.2 \mathrm{~Hz}, 1 \mathrm{H}, \mathrm{NH}), 7.63-7.58\left(\mathrm{~m}, 4 \mathrm{H}, H_{\text {arom}}\right)$, $7.45-7.40\left(\mathrm{~m}, 4 \mathrm{H}, H_{\text {arom }}\right), 7.31-7.18\left(\mathrm{~m}, 6 \mathrm{H}, H_{\text {arom }}\right), 5.46(\mathrm{~d}, J=5.3 \mathrm{~Hz}, 1 \mathrm{H}, \mathrm{OH}), 5.12(\mathrm{t}, J=$ $8.9 \mathrm{~Hz}, 1 \mathrm{H}, \mathrm{CHNH}), 4.93-4.89(\mathrm{~m}, 1 \mathrm{H}, \mathrm{CHOH}) \mathrm{ppm}$. 
${ }^{13}$ C-NMR $\left(100 \mathrm{MHz}, \mathrm{DMSO}-d_{6}\right) \delta=164.2(\mathrm{C}=\mathrm{O}), 143.5,141.1,133.6,131.1,131.1,129.3$, $129.3,128.3,128.3,127.6,127.6,127.5,127.5,127.0,126.8,126.8,126.7,124.7\left(18 \mathrm{x} \mathrm{C}_{\text {arom }}\right)$, 74.5 (C), 59.0 (C) ppm.

HRMS (ESI) $m / z$ : $(\mathrm{M}+\mathrm{H})^{+}$calc. for $\mathrm{C}_{21} \mathrm{H}_{18} \mathrm{BrNO}_{2}, 396.0594$ found 396.0600.

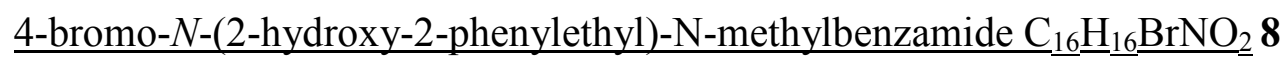

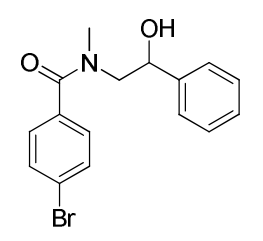

The titled compound was obtained with general procedure A in $80 \%$ yield, using general procedure B in $90 \%$ yield (acetonitrile) and $86 \%$ yield (DMF).

$\mathbf{M p}=133-135^{\circ} \mathrm{C}$.

${ }^{1}$ H-NMR $\left(500 \mathrm{MHz}, \mathrm{DMSO}-d_{6}\right) \delta=7.58\left(\mathrm{~d}, J=8.4 \mathrm{~Hz}, 2 \mathrm{H}, H_{\text {arom }}\right), 7.31(\mathrm{~d}, J=6.7 \mathrm{~Hz}, 4 \mathrm{H}$, $\left.H_{\text {arom }}\right), 7.27-7.22\left(\mathrm{~m}, 3 \mathrm{H}, H_{\text {arom }}\right), 5.22(\mathrm{~d}, J=4.6 \mathrm{~Hz}, 1 \mathrm{H}, \mathrm{OH}), 4.86(\mathrm{~s}, 1 \mathrm{H}, \mathrm{CHOH}), 3.52(\mathrm{~d}, J=$ $\left.7.5 \mathrm{~Hz}, 2 \mathrm{H}, \mathrm{CH}_{2}\right), 2.96\left(\mathrm{~s}, 3 \mathrm{H}, \mathrm{CH}_{3}\right) \mathrm{ppm}$.

${ }^{13}$ C-NMR (100 MHz, DMSO- $\left.d_{6}\right) \delta=170.0,169.2,143.5,143.0,131.2,131.0,129.2,128.9$, $128.1,127.2,126.0,125.8,122.5,122.1,70.42,69.7,58.0,55.12,33.18$ ppm.

HRMS (ESI) $m / z:(\mathrm{M}+\mathrm{H})^{+}$calc. for $\mathrm{C}_{16} \mathrm{H}_{16} \mathrm{BrNO}_{2} 334.0437$, found 334.0441.

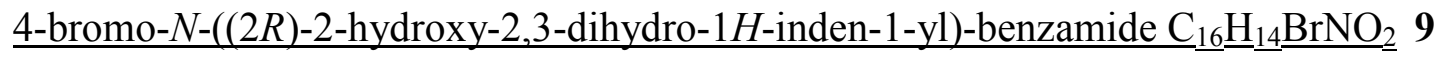

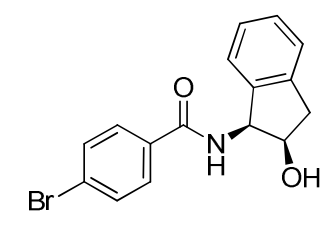

The titled compound was obtained with general procedure A in $96 \%$ yield, using general procedure B in 90\% yield (acetonitrile) and 96\% yield (DMF).

$\mathbf{M p}=186.5-187.5^{\circ} \mathrm{C}$. 
${ }^{1}$ H-NMR (400 MHz, DMSO- $\left.d_{6}\right) \delta=8.41(\mathrm{~d}, J=8.5 \mathrm{~Hz}, 1 \mathrm{H}, \mathrm{NH}), 7.92(\mathrm{~d}, J=8.5 \mathrm{~Hz}, 2 \mathrm{H}$, $\left.H_{\text {arom }}\right), 7.67\left(\mathrm{~d}, J=8.5 \mathrm{~Hz}, 2 \mathrm{H}, H_{\text {arom }}\right), 7.17-7.27\left(\mathrm{~m}, 4 \mathrm{H}, H_{\text {arom }}\right), 5.42-5.46(\mathrm{~m}, 1 \mathrm{H}, \mathrm{CHNH})$, $5.10(\mathrm{~d}, J=4.5 \mathrm{~Hz}, 1 \mathrm{H}, \mathrm{OH}), 4.50-4.54(\mathrm{~m}, 1 \mathrm{H}, \mathrm{CHOH}), 3.08-3.13\left(\mathrm{~m}, 1 \mathrm{H}, \mathrm{CH}_{2}\right), 2.91-2.86$ $\left(\mathrm{m}, 1 \mathrm{H}, \mathrm{CH}_{2}\right) \mathrm{ppm}$.

${ }^{13}$ C-NMR $\left(100 \mathrm{MHz}, \mathrm{DMSO}-d_{6}\right) \delta=165.8(\mathrm{C}=\mathrm{O}), 141.6,141.0,133.6,131.1,131.1,129.8$, 129.8, 127.4, 126.3, 124.9, 124.8, 124.4 (12 x C $\left.\mathrm{C}_{\text {arom }}\right), 72.1$ (C), 57.6 (C), 39.7 (C) ppm.

HRMS (ESI) $m / z:(\mathrm{M}+\mathrm{H})^{+}$calc. for $\mathrm{C}_{16} \mathrm{H}_{14} \mathrm{BrNO}_{2} 332.0281$, found 332.0282 .

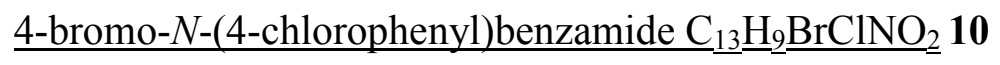<smiles>O=C(Nc1ccc(Cl)cc1)c1ccc(Br)cc1</smiles>

The titled compound was obtained with general procedure A in 95\% yield.

$\mathbf{M p}=219-220.5^{\circ} \mathrm{C}$.

${ }^{1}$ H-NMR $\left(400 \mathrm{MHz}, \mathrm{DMSO}-d_{6}\right) \delta=10.42(\mathrm{~s}, 1 \mathrm{H}, \mathrm{NH}), 7.90\left(\mathrm{~d}, J=8.6 \mathrm{~Hz}, 2 \mathrm{H}, H_{\text {arom. }}\right), 7.81$ (d, $\left.J=8.9 \mathrm{~Hz}, 2 \mathrm{H}, H_{\text {arom. }}\right), 7.75$ (d, $\left.J=8.6 \mathrm{~Hz}, 2 \mathrm{H}, H_{\text {arom. }}\right), 7.41$ (d, $\left.J=8.9 \mathrm{~Hz}, 2 \mathrm{H}, H_{\text {arom. }}\right) \mathrm{ppm}$.

${ }^{13}$ C-NMR (100 MHz, DMSO- $\left.d_{6}\right) \delta=164.6(\mathrm{C}=\mathrm{O}), 137.9,133.7,131.4,131.4,129.8,129.8$, $128.5,128.5,127.4,125.5,121.9121 .9\left(12 \times \mathrm{C}_{\text {arom }}\right) \mathrm{ppm}$.

HRMS (ESI) $m / z:(\mathrm{M}+\mathrm{H})^{+}$calc. for $\mathrm{C}_{13} \mathrm{H}_{9} \mathrm{BrClNO}_{2} 309.9629$, found 309.9626.

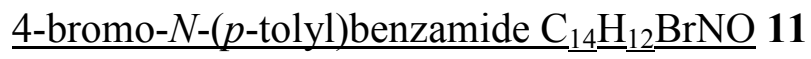<smiles>Cc1ccc(NC(=O)c2ccc(Br)cc2)cc1</smiles>

The titled compound was obtained with general procedure A in $94 \%$ yield.

$\mathbf{M p}=235.5-236.5^{\circ} \mathrm{C}$. 
${ }^{1}$ H-NMR (400 MHz, DMSO-d $\left.)_{6}\right) \delta=10.22(\mathrm{~s}, 1 \mathrm{H}, \mathrm{NH}), 7.90\left(\mathrm{~d}, J=8.5 \mathrm{~Hz}, 2 \mathrm{H}, H_{\text {arom. }}\right.$ ), 7.73 (d, $\left.J=8.5 \mathrm{~Hz}, 2 \mathrm{H} . H_{\text {arom. }}\right), 7.64\left(\mathrm{~d}, J=8.5 \mathrm{~Hz}, 2 \mathrm{H}, H_{\text {arom. }}\right), 7.15\left(\mathrm{~d}, J=8.5 \mathrm{~Hz}, 2 \mathrm{H}, H_{\text {arom. }}\right), 2.28(\mathrm{~s}$, $\left.3 \mathrm{H}, \mathrm{CH}_{3}\right) \mathrm{ppm}$.

${ }^{13}$ C-NMR (100 MHz, DMSO- $\left.d_{6}\right) \delta=164.3(\mathrm{C}=\mathrm{O}), 136.4,134.1,132.8,131.3,131.3,129.7$, 129.7, 129.0, 129.0, 125.2, 120.4, 120.4 (12 x $\left.\mathrm{C}_{\text {arom }}\right), 20.5$ (C) ppm.

HRMS (ESI) $m / z:(\mathrm{M}+\mathrm{H})^{+}$calc. for $\mathrm{C}_{14} \mathrm{H}_{12} \mathrm{BrNO} 290.0175$, found 290.0176.

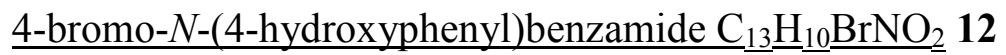

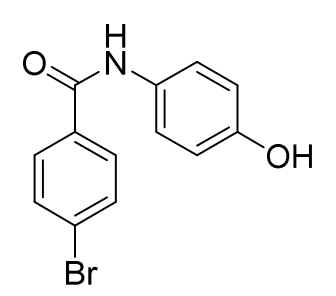

The titled compound was obtained with general procedure A in 50\% yield.

${ }^{1}$ H-NMR (400 MHz, DMSO-d 6 ) $\delta=10.09$ (s, 1H, RCONH), 9.28 (s, 1H, COH), 7.89 (d, J= 7.8 $\left.\mathrm{Hz}, 2 \mathrm{H}, H_{\text {arom }}\right), 7.72\left(\mathrm{~d}, J=7.8 \mathrm{~Hz}, 2 \mathrm{H}, H_{\text {arom }}\right), 7.52\left(\mathrm{~d}, J=8.3 \mathrm{~Hz}, 2 \mathrm{H}, H_{\text {arom }}\right), 6.74(\mathrm{~d}, 2 \mathrm{H}, J=$ $\left.8.3 \mathrm{~Hz}, H_{\text {arom }}\right)$ ppm.

${ }^{13}$ C-NMR $\left(100 \mathrm{MHz}, \mathrm{DMSO}-d_{6}\right) \delta=163.9(\mathrm{OCN}), 153.8,134.20,131.3,131.3,130.5,129.7$, $129.7,125.01,122.3,122.3,115.0,115.0\left(12 x C_{\text {arom }}\right)$ ppm.

HRMS (ESI) $m / z:(\mathrm{M}+\mathrm{H})^{+}$calc. for $\mathrm{C}_{13} \mathrm{H}_{10} \mathrm{BrNO}_{2}, 290.9895$ found 290.9968 .

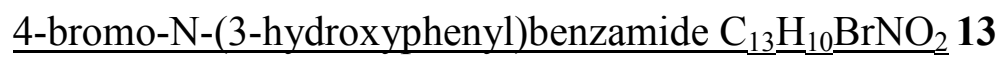<smiles>O=C(Nc1cccc(O)c1)c1ccc(Br)cc1</smiles>

The titled compound was obtained with general procedure A in $40 \%$ yield.

$$
\mathbf{M p}=213-215^{\circ} \mathrm{C}
$$


${ }^{1}$ H-NMR (400 MHz, DMSO-d 6 ) $\delta=10.18(\mathrm{~s}, 1 \mathrm{H}, \mathrm{RCONH}), 9.43(\mathrm{~s}, 1 \mathrm{H}, \mathrm{OH}), 7.89(\mathrm{~d}, J=7.9$ $\left.\mathrm{Hz}, 2 \mathrm{H}, H_{\text {arom }}\right), 7.72\left(\mathrm{~d}, J=7.9 \mathrm{~Hz}, 2 \mathrm{H}, H_{\text {arom }}\right), 7.34\left(\mathrm{~s}, 1 \mathrm{H}\right.$, Harom) $7.13\left(\mathrm{~m}, 2 \mathrm{H}, H_{\text {arom }}\right), 6.52$ (d, $\left.J=6.9 \mathrm{~Hz}, 1 \mathrm{H}, H_{\text {arom }}\right) \mathrm{ppm}$.

${ }^{13}$ C-NMR (100 MHz, DMSO- $\left.d_{6}\right) \delta=177.2(\mathrm{OCN}), 164.9,158.0,140.4,131.8,131.8,130.3$, 130.3, 129.7, 129.7, 125.7, 111.6, 100.0, 107.9 (12x Carom) ppm.

HRMS (ESI) $m / z:(\mathrm{M}+\mathrm{H})^{+}$calc. for $\mathrm{C}_{13} \mathrm{H}_{10} \mathrm{BrNO}_{2}, 290.9895$ found 290.9968.

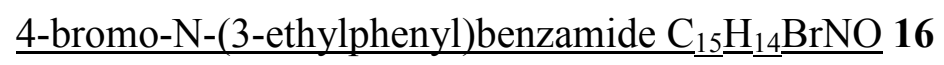<smiles>CCc1cccc(NC(=O)c2ccc(Br)cc2)c1</smiles>

The titled compound was obtained with general procedure A.

${ }^{1}$ H-NMR (400 MHz, DMSO-d $\left.{ }_{6}\right) \delta=10.25(\mathrm{~s}, 1 \mathrm{H}, \mathrm{NH}), 7.93\left(\mathrm{~d}, J=8.5 \mathrm{~Hz}, 2 \mathrm{H}, H_{\text {arom }}\right), 7.76(\mathrm{~d}, J$ $\left.=8.5 \mathrm{~Hz}, 2 \mathrm{H}, H_{\text {arom }}\right), 7.60\left(\mathrm{~m}, 2 \mathrm{H}, H_{\text {arom }}\right), 7.26\left(\mathrm{t}, J=7.8 \mathrm{~Hz}, 1 \mathrm{H}, H_{\text {arom }}\right), 6,97(\mathrm{~d}, J=7.8 \mathrm{~Hz}, 1 \mathrm{H}$, $\mathrm{H}_{\text {arom }}$ ), 2.61 (q, $\left.J=8 \mathrm{~Hz}, 3 \mathrm{H}, \mathrm{CH}_{2}\right), 1.20\left(\mathrm{t}, J=8 \mathrm{~Hz}, 2 \mathrm{H}, \mathrm{CH}_{3}\right) \mathrm{ppm}$.

${ }^{13}$ C-NMR (100 MHz, DMSO- $\left.d_{6}\right) \delta=164.9$ (NCO) 144.6, 139.4, 134.5, 131.8, 131.8, 130.23, $130.23,129,0,125.7,123.8,120.2,118.3$ (12x $\left.C_{\text {arom }}\right), 28.8(C), 16.01(C)$ ppm.

HRMS (ESI) $m / z:(\mathrm{M}+\mathrm{H})^{+}$calc. for $\mathrm{C}_{15} \mathrm{H}_{14} \mathrm{BrNO}, 303.0259$ found 303.0336 .

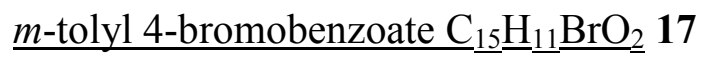<smiles>Cc1cccc(OC(=O)c2ccc(Br)cc2)c1</smiles>

The titled compound was obtained with general procedure A. 
${ }^{1}$ H-NMR $\left(400 \mathrm{MHz}, \mathrm{DMSO}_{-} \mathrm{d}_{6}\right) \delta=8.06\left(\mathrm{~d}, J=8.7 \mathrm{~Hz}, 2 \mathrm{H}, H_{\mathrm{arom}}\right), 7.86(\mathrm{~d}, J=8.7 \mathrm{~Hz}, 2 \mathrm{H}$, $\left.H_{\text {arom }}\right), 7.36\left(\mathrm{t}, J=7.7 \mathrm{~Hz}, 1 \mathrm{H}, H_{\text {arom }}\right), 7.15-7.04\left(\mathrm{~m}, 3 \mathrm{H}, H_{\text {arom }}\right), 2.35\left(\mathrm{~s}, 3 \mathrm{H}, \mathrm{CH}_{3}\right) \mathrm{ppm}$.

${ }^{13}$ C-NMR (100 MHz, DMSO- $\left.d_{6}\right) \delta=164.4$ (OCO) 150.9, 139.8, 132.6 ,132.6, 132.15, 132.15, $131.74,129.8,128.7,127.2,122.7,119.3$ (12x $\left.C_{\text {arom }}\right), 21,27(C)$ ppm.

HRMS (ESI) $m / z:(\mathrm{M}+\mathrm{H})^{+}$calc. for $\mathrm{C}_{15} \mathrm{H}_{11} \mathrm{BrO}_{2}, 289.9942$ found 290.0015 .

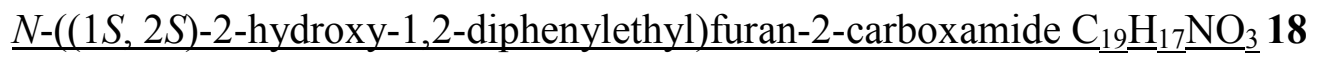<smiles>O=C(N[C@H](c1ccccc1)[C@H](O)c1ccccc1)c1ccco1</smiles>

The titled compound was obtained with general procedure A in $92 \%$ yield.

$\mathbf{M p}=214.5-216^{\circ} \mathrm{C}$.

${ }^{1}$ H-NMR $\left(400 \mathrm{MHz}, \mathrm{DMSO}-d_{6}\right) \delta=8.45(\mathrm{~d}, J=9.1 \mathrm{~Hz}, 1 \mathrm{H}, \mathrm{NH}), 7.78\left(\mathrm{~s}, 1 \mathrm{H}, H_{\text {arom. }}\right), 7.38$ $7.35\left(\mathrm{~m}, 4 \mathrm{H}, H_{\text {arom. }}\right), 7.28-7.18\left(\mathrm{~m}, 6 \mathrm{H}, H_{\text {arom }}\right), 7.00\left(\mathrm{~d}, 1 \mathrm{H}, J=3.9 \mathrm{~Hz}, H_{\text {arom. }}\right), 6.57-6.56(\mathrm{~m}$, $1 \mathrm{H}, H_{\text {arom. }}$ ), 5.49 (d, $\left.J=5.1 \mathrm{~Hz}, 1 \mathrm{H}, \mathrm{OH}\right), 5.09$ (t, $\left.J=8.5 \mathrm{~Hz}, 1 \mathrm{H}, \mathrm{CHNH}\right), 4.97-4.94(\mathrm{~m}, 1 \mathrm{H}$, $\mathrm{CHOH}) \mathrm{ppm}$.

${ }^{13}$ C-NMR $\left(100 \mathrm{MHz}, \mathrm{DMSO}-d_{6}\right) \delta=156.5(\mathrm{C}=\mathrm{O}), 147.6,144.9,143.2,140.8,128.3,128.3$, $127.6,127.6,127.5,127.5,127.0,126.8,126.8,126.7,113.5,111.8,\left(16 \times \mathrm{C}_{\text {arom. }}\right) 74.2(\mathrm{C}), 58.3$ (C) ppm.

HRMS (ESI) $m / z:(\mathrm{M}+\mathrm{H})^{+}$calc. for $\mathrm{C}_{19} \mathrm{H}_{17} \mathrm{NO}_{3} 308.1281$, found 308.1282.

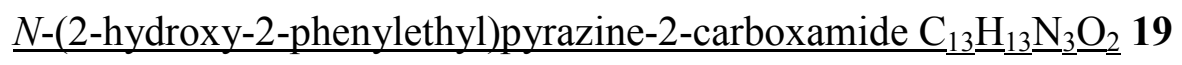


<smiles>O=C(NCC(O)c1ccccc1)c1cnccn1</smiles>

The titled compound was obtained with general procedure $\mathrm{C}$ in $95 \%$ yield.

$\mathbf{M p}=121.5-123^{\circ} \mathrm{C}$.

${ }^{1}$ H-NMR (400 MHz, DMSO- $\left.d_{6}\right) \delta=9.18(\mathrm{~d}, J=1.3 \mathrm{~Hz}, 1 \mathrm{H}, \mathrm{NH}), 8.87(\mathrm{~d}, J=2.4 \mathrm{~Hz}, 1 \mathrm{H}$, $\left.H_{\text {arom }}\right), 8.74-8.68\left(\mathrm{~m}, 2 \mathrm{H}, H_{\text {arom }}\right), 7.40-7.31\left(\mathrm{~m}, 4 \mathrm{H}, H_{\text {arom }}\right), 7.27-7.23\left(\mathrm{~m}, 1 \mathrm{H}, H_{\text {arom }}\right), 5.61(\mathrm{~d}$, $J=4.5 \mathrm{~Hz}, 1 \mathrm{H}, \mathrm{OH}), 4.84-4.80(\mathrm{~m}, 1 \mathrm{H}, \mathrm{CH}), 3.63-3.57\left(\mathrm{~m}, 1 \mathrm{H}, \mathrm{CH}_{2}\right), 3.46-3.39(\mathrm{~m}, 1 \mathrm{H}$, $\left.\mathrm{CH}_{2}\right)$ ppm.

${ }^{13}$ C-NMR $\left(100 \mathrm{MHz}, \mathrm{DMSO}-d_{6}\right) \delta=162.6(\mathrm{C}=\mathrm{O}), 147.6,144.5,143.4,143.3,143.3,128.1$, 128.1, 127.1, 125.9, 125.9, 70.9 (C), 46.8 (C) ppm.

HRMS (ESI) $m / z:(\mathrm{M}+\mathrm{H})^{+}$calc. for $\mathrm{C}_{13} \mathrm{H}_{13} \mathrm{~N}_{3} \mathrm{O}_{2} 244.1081$, found 244.1082.

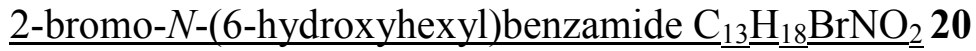<smiles>O=C(NCCCCCCO)c1ccccc1Br</smiles>

The titled compound was obtained with general procedure C in TPGS-750-M in 77\% yield.

$\mathbf{M p}=63-65^{\circ} \mathrm{C}$.

${ }^{1}$ H-NMR $\left(400 \mathrm{MHz}, \mathrm{DMSO}-d_{6}\right) \delta=8.36(\mathrm{t}, J=5.2 \mathrm{~Hz}, 1 \mathrm{H}, \mathrm{NH}), 7.63\left(\mathrm{~d}, J=8.0 \mathrm{~Hz}, 1 \mathrm{H}, H_{\text {arom }}\right)$ $7.44-7.40\left(\mathrm{~m}, 1 \mathrm{H}, H_{\text {arom }}\right), 7.36-7.32\left(\mathrm{~m}, 2 \mathrm{H}, H_{\text {arom }}\right), 4.33(\mathrm{t}, J=5.2 \mathrm{~Hz}, 1 \mathrm{H}, \mathrm{OH}), 3.39(\mathrm{q}, J=$ $\left.6.5 \mathrm{~Hz}, 2 \mathrm{H}, \mathrm{CH}_{2}\right), 3.20$ (q, $\left.J=6.5 \mathrm{~Hz}, 2 \mathrm{H}, \mathrm{CH}_{2}\right), 1.52-1.32\left(\mathrm{~m}, 8 \mathrm{H}, 4\right.$ x $\left.\mathrm{CH}_{2}\right) \mathrm{ppm}$.

${ }^{13}$ C-NMR (100 MHz, DMSO- $\left.d_{6}\right) \delta=167.0(\mathrm{C}=\mathrm{O}), 139.5,132.6,130.6,128.7,127.5,118.9$ (6x $\mathrm{C}_{\text {arom}}$ ), $60.7(\mathrm{C}), 38.9(\mathrm{C}), 32.5(\mathrm{C}), 28.9$ (C), $26.3(\mathrm{C}), 25.2(\mathrm{C}) \mathrm{ppm}$.

HRMS (ESI) $m / z:(\mathrm{M}+\mathrm{H})^{+}$calc. for $\mathrm{C}_{13} \mathrm{H}_{18} \mathrm{BrNO}_{2} 300.0594$, found 300.0595 . 
<smiles>O=C(NCC(O)c1ccccc1)c1ccccc1Br</smiles>

The titled compound was obtained with general procedure A in $63 \%$ yield.

$\mathbf{M p}=116-117^{\circ} \mathrm{C}$.

${ }^{1}$ H-NMR $\left(400 \mathrm{MHz}, \mathrm{DMSO}-d_{6}\right) \delta=8.42(\mathrm{t}, J=5.6 \mathrm{~Hz}, 1 \mathrm{H}, \mathrm{NH}), 7.62(\mathrm{dd}, J=7.9,1.1 \mathrm{~Hz}, 1 \mathrm{H}$, $\left.H_{\text {arom }}\right), 7.42-7.24\left(\mathrm{~m}, 8 \mathrm{H}, H_{\text {arom }}\right), 5.47(\mathrm{~d}, J=4.5 \mathrm{~Hz}, 1 \mathrm{H}, \mathrm{OH}), 4.77-4.73(\mathrm{~m}, 1 \mathrm{H}, \mathrm{CHNH})$, $3.47-3.34\left(\mathrm{~m}, 2 \mathrm{H}, \mathrm{CH}_{2}\right) \mathrm{ppm}$.

${ }^{13}$ C-NMR $\left(100 \mathrm{MHz}, \mathrm{DMSO}-d_{6}\right) \delta=167.2(\mathrm{C}=\mathrm{O}), 143.6,139.0,132.6,130.7,128.8,128.0$, 128.0, 127.4, 127.1, 126.2, 126.2, 118.9, (12x C $\mathrm{arom}_{\text {), }} 71.1(\mathrm{C}), 47.1$ (C) ppm.

HRMS (ESI) $m / z:(\mathrm{M}+\mathrm{H})^{+}$calc. for $\mathrm{C}_{15} \mathrm{H}_{14} \mathrm{BrNO}_{2} 320.0281$, found 320.0281 .

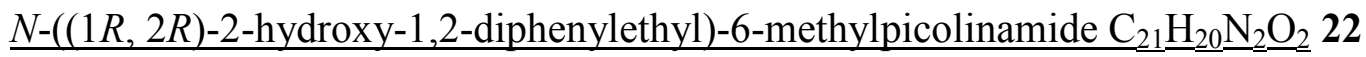<smiles>Cc1cccc(C(=O)N[C@H](c2ccccc2)[C@H](O)c2ccccc2)n1</smiles>

The titled compound was obtained with general procedure A in $93 \%$ yield.

$\mathbf{M p}=126-128^{\circ} \mathrm{C}$.

${ }^{1}$ H-NMR (400 MHz, DMSO- $\left.d_{6}\right) \delta=8.98(\mathrm{~d}, J=8.9 \mathrm{~Hz}, 1 \mathrm{H}, \mathrm{NH}), 7.85(\mathrm{t}, J=7.7 \mathrm{~Hz}, 1 \mathrm{H}$, $\left.H_{\text {arom. }}\right), 7.76$ (d, $\left.J=7.5 \mathrm{~Hz}, 1 \mathrm{H}, H_{\text {arom. }}\right), 7.47$ (d, $\left.J=7.5 \mathrm{~Hz}, 1 \mathrm{H}, H_{\text {arom. }}\right), 7.14-7.25$ (m, 10H, $\left.H_{\text {arom. }}\right), 5.84(\mathrm{~d}, J=4.3 \mathrm{~Hz}, 1 \mathrm{H}, \mathrm{OH}), 5.18-5.22(\mathrm{~m}, 1 \mathrm{H}, \mathrm{CHNH}), 5.11-5.13(\mathrm{~m}, 1 \mathrm{H}, \mathrm{CHOH})$, $2.58\left(\mathrm{~s}, 3 \mathrm{H}, \mathrm{CH}_{3}\right) \mathrm{ppm}$.

${ }^{13}$ C-NMR (100 MHz, DMSO- $\left.d_{6}\right) \delta=162.7(\mathrm{C}=\mathrm{O}), 157.1,148.9,141.8,139.4,138.0,127.9$, $127.9,127.5,127.5,127.5,127.5,127.1,126.8,126.6,126.6,126.2,118.8$ (17 x $\left.\mathrm{C}_{\text {arom }}\right), 74.5$ (C), 58.4 (C), 24.0 (C) ppm. 
HRMS (ESI) $m / z:(\mathrm{M}+\mathrm{H})^{+}$calc. for $\mathrm{C}_{21} \mathrm{H}_{20} \mathrm{~N}_{2} \mathrm{O}_{2} 333.1598$, found 333.1602 .

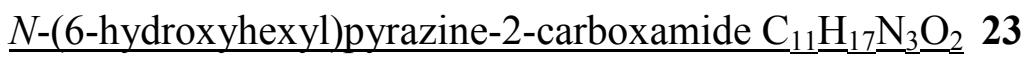<smiles>O=C(NCCCCCCO)c1cnccn1</smiles>

The titled compound was obtained with general procedure $\mathrm{C}$ in $85 \%$ yield.

$\mathbf{M p}=89-90.5^{\circ} \mathrm{C}$.

${ }^{1}$ H-NMR $\left(400 \mathrm{MHz}\right.$, DMSO- $\left.d_{6}\right) \delta=9.17(\mathrm{~d}, J=1.5 \mathrm{~Hz}, 1 \mathrm{H}, \mathrm{NH}), 8.93(\mathrm{t}, J=6.1 \mathrm{~Hz}, 1 \mathrm{H}$, $\left.H_{\text {arom }}\right), 8.87\left(\mathrm{~d}, J=2.5 \mathrm{~Hz}, 1 \mathrm{H}, H_{\text {arom }}\right), 8.73\left(\mathrm{~m}, 1 \mathrm{H}, H_{\text {arom }}\right), 4.26(\mathrm{bs}, 1 \mathrm{H}, \mathrm{OH}), 3.36(\mathrm{t}, J=6.2 \mathrm{~Hz}$, 2H, $\mathrm{CH}_{2}$ ), 3.29 (q, $J=6.9 \mathrm{~Hz}, 2 \mathrm{H}, \mathrm{CH}_{2}$ ), 1.53 (qi, $J=6.7 \mathrm{~Hz}, 2 \mathrm{H}, \mathrm{CH}_{2}$ ), $1.43-1.36(\mathrm{~m}, 2 \mathrm{H}$, $\left.\mathrm{CH}_{2}\right), 1.278-1.27\left(\mathrm{~m}, 4 \mathrm{H}, 2 \times \mathrm{CH}_{2}\right) \mathrm{ppm}$.

${ }^{13}$ C-NMR $\left(100 \mathrm{MHz}, \mathrm{DMSO}-d_{6}\right) \delta=162.7(\mathrm{C}=\mathrm{O}), 147.3,145.0,143.5,143.2\left(4 \times \mathrm{C}_{\text {arom }}\right), 60.7$ (C), 38.8 (C), 32.5 (C), 29.1 (C), 26.4 (C), 25.3 ppm.

HRMS (ESI) $m / z:(\mathrm{M}+\mathrm{H})^{+}$calc. for $\mathrm{C}_{11} \mathrm{H}_{17} \mathrm{~N}_{3} \mathrm{O}_{2} 224.1394$, found 224.1397 .

2-chloro- $N$-(2-hydroxy-2-phenylethyl)nicotinamide $\mathrm{C}_{1} \underline{1} \underline{H}_{13} \underline{\mathrm{ClN}_{2}} \underline{\mathrm{O}_{2}} 24$<smiles>O=C(NCC(O)c1ccccc1)c1cccnc1Cl</smiles>

The titled compound was obtained with general procedure $\mathrm{C}$ in $85 \%$ yield.

$\mathbf{M p}=120-122^{\circ} \mathrm{C}$.

${ }^{1}$ H-NMR (400 MHz, DMSO-d 6 ) $\delta=8.68(\mathrm{t}, J=5.9 \mathrm{~Hz}, 1 \mathrm{H}, \mathrm{RCON} H), 8.45(\mathrm{dd}, J=7.8 \mathrm{~Hz}, 2.0$ $\left.\mathrm{Hz}, 1 \mathrm{H}, H_{\text {arom }}\right), 7.80$ (dd, $\left.J=7.5 \mathrm{~Hz}, 2.2 \mathrm{~Hz}, 1 \mathrm{H}, H_{\text {arom }}\right), 7.48$ (dd, $J=7.8 \mathrm{~Hz}, 4.8 \mathrm{~Hz}, 1 \mathrm{H}, H_{\text {arom }}$ ), $7.42-7.34\left(\mathrm{~m}, 4 \mathrm{H}, H_{\text {arom }}\right), 7.27\left(\mathrm{~m}, 1 \mathrm{H}, H_{\text {arom }}\right), 5.55(\mathrm{~d}, J=4.6 \mathrm{~Hz}, 1 \mathrm{H}, \mathrm{CH}), 3.51-3.45(\mathrm{~m}, 1 \mathrm{H}$, $\left.\mathrm{CH}_{2}\right), 3.40-3.33\left(\mathrm{~m}, 1 \mathrm{H}, \mathrm{CH}_{2}\right) \mathrm{ppm}$.

${ }^{13}$ C-NMR $\left(100 \mathrm{MHz}, \mathrm{DMSO}-d_{6}\right) \delta=165.6(\mathrm{OCN}), 150.5,147.0,144.0,138.5,133.6,128.5$, 128.5, 127.6, 126.7, 126.7, $123.4\left(11 \mathrm{x} C_{\text {arom }}\right), 71.6(\mathrm{COH}), 47.6\left(\mathrm{CNH}_{2}\right) \mathrm{ppm}$. 
HRMS (ESI) $m / z:(\mathrm{M}+\mathrm{H})^{+}$calc. for $\mathrm{C}_{14} \mathrm{H}_{13} \mathrm{ClN}_{2} \mathrm{O}_{2}, 276.0666$ found 276.0738 .

(R)-N-(2-hydroxy-1-phenylethyl)isobutyramide $\mathrm{C}_{12} \underline{\mathrm{H}_{17}} \underline{\mathrm{NO}_{2}} 25$<smiles>CC(C)C(=O)NC(CO)c1ccccc1</smiles>

The titled compound was obtained with general procedure $\mathrm{C}$ in $81 \%$ yield.

$\mathbf{M p}=185.5-120.5^{\circ} \mathrm{C}$.

${ }^{1}$ H-NMR $\left(400 \mathrm{MHz}\right.$, DMSO-d $\left.\mathrm{d}_{6}\right) \delta=8.09(\mathrm{~d}, J=8.6 \mathrm{~Hz}, 1 \mathrm{H}, \mathrm{NH}), 7.36-7.20\left(\mathrm{~m}, 5 \mathrm{H}, H_{\text {arom }}\right)$, $7.22\left(\mathrm{~m}, 1 \mathrm{H}, H_{\text {arom }}\right), 4.84-4.79\left(\mathrm{~m}, 2 \mathrm{H}, \mathrm{OH}, \mathrm{CH}_{3} \mathrm{CH}\right), 3.54\left(\mathrm{~d}, J=5.2 \mathrm{~Hz}, 2 \mathrm{H}, \mathrm{CH}_{2}\right), 2.49(\mathrm{~m}$, $1 \mathrm{H}, \mathrm{C} H \mathrm{NH}), 1.11\left(\mathrm{~d}, J=7.0 \mathrm{~Hz}, 3 \mathrm{H}, \mathrm{CH}_{3}\right), 0.98\left(\mathrm{~d}, J=7.0 \mathrm{~Hz}, 3 \mathrm{H}, \mathrm{CH}_{3}\right) \mathrm{ppm}$.

${ }^{13}$ C-NMR (100 MHz, DMSO-d 6 ) $\delta=175.7$ (C), 141.6, 128.0, 128.0, 126.8, 126.6, $126.7(6 \mathrm{x}$ $\left.\mathrm{C}_{\text {arom }}\right), 64.7$ (C), 54.7 (C), 33.9 (C), 19.7 (C), 19.6 (C) ppm.

HRMS (ESI) $m / z:(\mathrm{M}+\mathrm{H})^{+}$calc. for $\mathrm{C}_{12} \mathrm{H}_{17} \mathrm{NO}_{2}, 207.1259$ found 208.1332 .

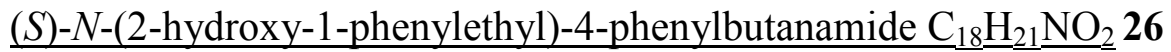<smiles>O=C(CCCc1ccccc1)N[C@H](CO)c1ccccc1</smiles>

The titled compound was obtained with general procedure A in $98 \%$ yield.

$\mathbf{M p}=92.5-94^{\circ} \mathrm{C}$.

${ }^{1}$ H-NMR (400 MHz, DMSO-d $\left.d_{6}\right) \delta=8.18(\mathrm{~d}, J=8.2 \mathrm{~Hz}, 1 \mathrm{H}, \mathrm{NH}), 7.31-7.15\left(\mathrm{~m}, 10 \mathrm{H}, H_{\text {arom. }}\right.$ ), $4.89-4.81(\mathrm{~m}, 2 \mathrm{H}, \mathrm{C} H \mathrm{NH}, \mathrm{OH}), 3.56-3.52\left(\mathrm{~m}, 2 \mathrm{H}, \mathrm{CH}_{2}\right), 2.55\left(\mathrm{t}, J=7.6 \mathrm{~Hz}, 2 \mathrm{H}, \mathrm{CH}_{2}\right), 2.17$ (t, $\left.J=7.6 \mathrm{~Hz}, 2 \mathrm{H}, \mathrm{CH}_{2}\right), 1.80\left(\mathrm{qi}, J=7.6 \mathrm{~Hz}, 2 \mathrm{H}, \mathrm{CH}_{2}\right) \mathrm{ppm}$.

${ }^{13}$ C-NMR $\left(100 \mathrm{MHz}, \mathrm{DMSO}-d_{6}\right) \delta=171.5(\mathrm{C}=\mathrm{O}), 141.8,141.5,128.3,128.3,128.3,128.3$, 128.0, 128.0, 126.9, 126.9, 126.7 ,125.7 (12 x C arom), 64.7 (C), 54.9 (C), 34.9 (C), 34.7 (C), 27.2 (C) ppm.

HRMS (ESI) $m / z:(\mathrm{M}+\mathrm{H})^{+}$calc. for $\mathrm{C}_{18} \mathrm{H}_{21} \mathrm{NO}_{2} 284.1645$, found 284.1650 . 


\section{References}

1. D. J. Upadhyaya, A. Barge, R. Stefania, G. Cravotto, Tetrahedron Lett. 2007, 48, 83188322.

2. M. Arifuddin, N. Lakshmikant, N. Rajasekar, D. B. Shinde, Indian J. Chem. - Sect. B Org. Med. Chem. 2012, 51, 1168-1172.

3. D. M. Shendage, R. Fröhlich, G. Haufe, Org. Lett. 2004, 6, 3675-3678. boc

4. H. Nakatsuji, M. Morimoto, T. Misaki, Y. Tanabe, Tetrahedron 2007, 63, 12071-12080. 